\title{
Corrosión de la aleación 8090 en medios salinos de agresividad moderada
}

\author{
A. Conde ${ }^{(*)}$ y J.J. de Damborenea ${ }^{(* *)}$
}

\begin{abstract}
Resumen A pesar del alto grado de conocimiento existente sobre las propiedades mecánicas de las aleaciones Al-Li, los trabajos sobre su comportamiento frente a la corrosión son más bien escasos. En el presente trabajo, se pretende caracterizar, mediante la espectroscopía de impedancia electroquímica, el comportamiento frente a la corrosión de la aleación 8090 en un medio de agresividad moderada, como es el $\mathrm{NaCl}$ 0,06 M. Al final del ensayo $(100 \mathrm{~h})$ la probeta mostraba sólo un ligero ataque intergranular. Esta situación se pone de manifiesto en la evolución de los diagramas de impedancia. Durante las primeras $50 \mathrm{~h}$ no se aprecian desviaciones importantes con respecto al comportamiento ideal descrito por el circuito de Randles. Con el tiempo, el diagrama de impedancia está constituido por dos arcos capacitivos. Mientras el semicírculo correspondiente a las altas frecuencias podría ser consecuencia de la transferencia de carga y migración iónica a través de la capa de óxido, el semicírculo de las más bajas frecuencias podría deberśe a la adsorción de los aniones procedentes del electrólito.
\end{abstract}

Palabras clave: Corrosión. Aleaciones Al-Li. Impedancia electroquímica.

\section{Corrosion behaviour of 8090 alloy in saline solution with moderate aggressiveness}

\begin{abstract}
Corrosion studies of Al-Li alloys are not so extensive and concentrate almost exclusively on atmospheric exposure tests and accelerated laboratory tests due to the fact they provide a reasonable approximation to the real behaviour of the alloy in service conditions. This paper attempts to establish a correlation between the evolution of the impedance diagrams and the process of the attack undergone by a commercial 8090 T8171 alloy, with the aim of establishing the kinetics of the corrosion process. After $100 \mathrm{~h}$ of immersion, samples showed only a slight intergranular attack. As a result of the low aggressiveness of the solution no major deviations from the ideal behaviour described by the Randles circuit are expected in the impedance plots. After 50 hours of testing, the impedance diagram evolves towards two semicircles which seem to be related with the charge transfer and ionic migration through the oxide layer and the adsorption of electrolytre anions.
\end{abstract}

Keywords: Corrosion. Al-Li alloys. Electrochemical impedance.

\section{INTRODUCCIÓN}

La utilización del aluminio y sus aleaciones ocupa, desde hace más de un cuarto de siglo, un lugar destacado en la industria del transporte y de la automoción. Durante la década de los 80 se creyó que la irrupción de nuevos materiales, compuestos metálicos y/o cerámicos supondría, el punto final en la expansión del aluminio. Sin embargo, en los últimos años se está asistiendo a un progresivo

(*) University College London. Chemistry Dept. 20 Gordon Street WC1H 0AJ. Londres (R.U.)

(**) Centro Nacional de Investigaciones Metalúrgicas, CENIM (CSIC), Avda. de Gregorio del Amo 8. 28040-Madrid (España). incremento de las aplicaciones de este tipo de aleaciones junto al desarrollo y búsqueda de otras nuevas con mejores propiedades mecánicas y de resistencia a la corrosión (1).

Un claro ejemplo de esta evolución, lo constituyen las aleaciones de Al-Li. Desde que en el período 1984-1987, se hiciera pública la patente de la 8090 , el interés por este tipo de aleaciones ha vuelto a surgir por sus especiales características de baja densidad y alto módulo elástico.

A pesar del alto grado de conocimiento existente sobre las propiedades mecánicas de estas aleaciones, los trabajos sobre su comportamiento frente a la corrosión son más bien escasos, centrándose casi exclusivamente en ensayos acelerados según 
normas y en la exposición atmosférica. En el presente trabajo se, pretende caracterizar, mediante la espectroscopía de impedancia electroquímica, el comportamiento frente a la corrosión de la aleación 8090 en un medio de agresividad moderada, como es el $\mathrm{NaCl} 0,06 M$.

\section{PROCEDIMIENTO EXPERIMENTAL}

La aleación objeto de estudio está compuesta básicamente por aluminio y litio, fabricada en forma de planchas de $50 \mathrm{~mm}$ de espesor, $1.000 \mathrm{~mm}$ de longitud y $1.000 \mathrm{~mm}$ de anchura y su composición es la siguiente:

$\mathrm{Al}-2,4 \% \mathrm{Li}-1,15 \% \mathrm{Cu}-0,67 \% \mathrm{Mg}-$ $-0,11 \% \mathrm{Zr}-0,05 \% \mathrm{Fe}-0,02 \% \mathrm{Si}-0,0004 \% \mathrm{Na}$

Fue suministrada en estado T8171. Este tratamiento consistió en un proceso de solubilización y temple, con una deformación plástica en frío del $6 \%$ seguida de un envejecimiento durante $10 \mathrm{~h}$ a $150{ }^{\circ} \mathrm{C}$, que define un estado de envejecimiento próximo al pico de maduración.

Las medidas electroquímicas se llevaron a cabo mediante un sistema convencional de tres electrodos. Se utilizó como referencia un electrodo de calomelanos saturado y, para cerrar el circuito, un contraelectrodo de platino. Por último, el electrodo de trabajo esta constituido por $1 \mathrm{~cm}^{2}$ de la aleación objeto de estudio.

Los electrodos se conectaron a un potenciostato y a un analizador de respuesta en frecuencia y las operaciones de adquisición y análisis de los datos obtenidos se realizaron de manera automática mediante un programa adecuado. La señal de perturbación aplicada fue de $10 \mathrm{mV}$ de amplitud y el barrido de frecuencias, de $50 \mathrm{kHz}$ a $10 \mathrm{mHz}$. Para las simulaciones matemáticas de la impedancia, se utilizó el programa Equivcrt de B. Boukamp (2). Las primeras medidas se realizaron tras $6 \mathrm{~h}$ de inmersión de la probeta en la solución y luego se repitieron a intervalos de $24 \mathrm{~h}$, hasta completar el período total de inmersión de $100 \mathrm{~h}$.

El electrólito empleado fue $\mathrm{NaCl} 0,06 M$ preparado a partir de reactivo químicamente puro. $\mathrm{La}$ solución de ensayo tenía pH neutro.

\section{RESULTADOS Y DISCUSIÓN}

La observación macroscópica realizada al finalizar el ensayo no mostraba signos externos de ataque apreciables (picaduras, ampollas...), aunque el análisis metalográfico de la misma revelaba, a las $100 \mathrm{~h}$, un incipiente ataque intergranular, tal y

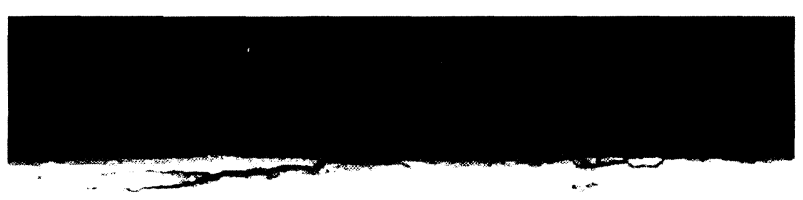

FIG. 1.- Ataque intergranular detectado tras $100 \mathrm{~h}$ de inmersión.

FIG. 1.- Intergranular attack after $100 \mathrm{~h}$ of testing.

como puede apreciarse en la figura $1 . \mathrm{Al} \mathrm{pH}$ de la disolución experimental, la capa de óxido protectora que de forma natural se genera sobre el aluminio $\mathrm{y}$, sus aleaciones, es estable y aunque la presencia de cloruros altera su estabilidad, la baja concentración de los mismos existente en la solución hace que la resistencia de dicha película protectora sea mayor que en otros medios más agresivos (3).

Esta situación queda perfectamente reflejada en los diagramas de impedancia realizados a lo largo del ensayo. Durante las primeras $50 \mathrm{~h}$ no se aprecian desviaciones importantes con respecto al comportamiento ideal descrito por el circuito de Randles. En la figura 2 se recoge el diagrama representativo de lo obtenido hasta las primeras 50 h. de ensayo. En él, se observa que el diagrama de Nyquist está constituido por un primer semicírculo capacitivo que se encuentra ligeramente deprimido, seguido a frecuencias bajas por un arco inductivo. Con el tiempo, este comportamiento inductivo desaparece, dando lugar a otro semicírculo capacitivo, como se muestra en la figura 3 para las $100 \mathrm{~h}$ de ensayo.

Como se puede apreciar, hay una depresión del arco capacitivo en las altas frecuencias, constatándose que el origen del semicírculo se sitúa por debajo del eje real de la impedancia. Sin embargo, esta depresión se hace más patente en la representación de Bode, donde la pendiente de la recta descrita en el intervalo de frecuencias asociada al comportamiento capacitivo, es ligeramente inferior a la unidad. En un sistema bajo control de activación, dicha recta forma un ángulo de $45^{\circ}$ con el eje de la frecuencia. Desviaciones de dicho valor pueden indicar que otros procesos, además del de transferencia de carga, pueden estar teniendo lugar en la intercara metal/electrólito. Sin embargo, en este caso, la pendiente de la recta en el diagrama de Bode se mantiene constante durante todo el ensayo y tampoco aparecen cambios en la representación 

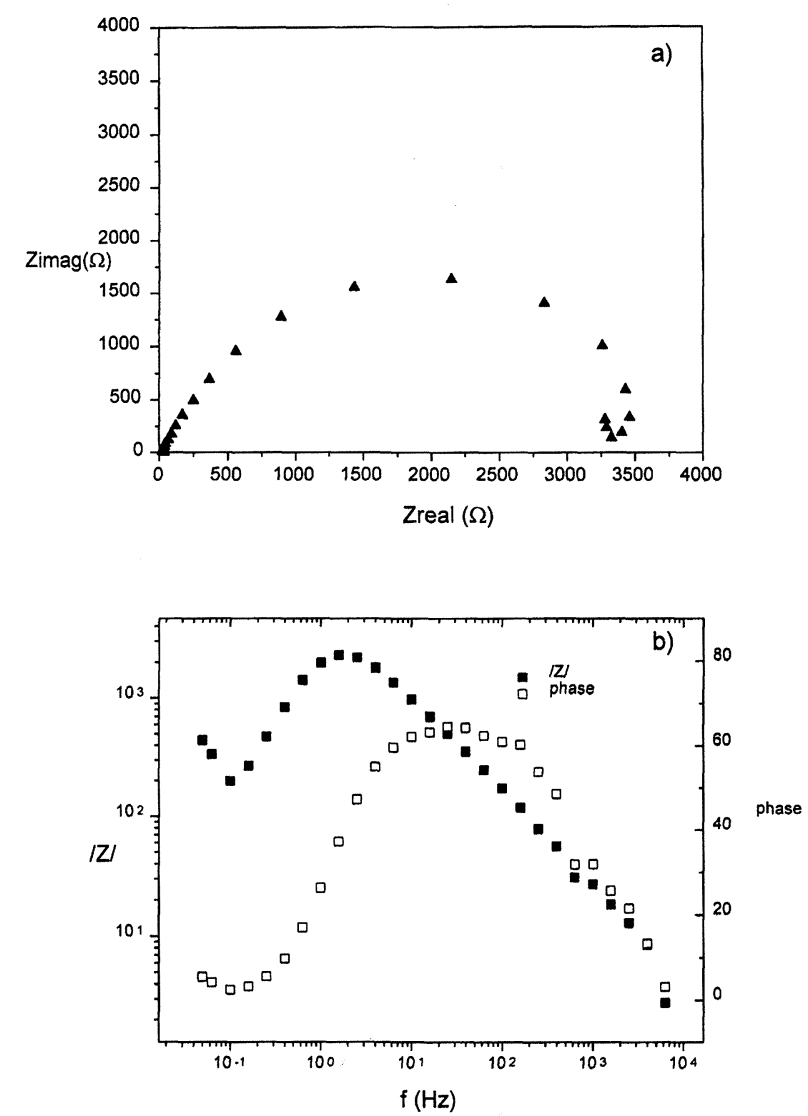

FIG. 2.- Diagramas de $a$ ) Nyquist y $b$ ) Bode, correspondientes a $50 \mathrm{~h}$ de inmersión.

FIG. 2.- Impedance diagram a) Nyquist and b) Bode after $50 \mathrm{~h}$ of testing.

de Bode del ángulo. Tanto la altura y la anchura del máximo, como su localización en el intervalo de frecuencias permanece invariable. Por tanto, la disminución de la pendiente en el diagrama de Bode parece estar originada por una dispersión de la constante capacitiva de tiempo provocada por una falta de homogeneidad superficial. De este modo, se puede relacionar el arco de las altas frecuencias con el mecanismo de transferencia de carga que tiene lugar a través de la capa de óxido.

En las bajas frecuencias, se describe lo que parece ser un segundo semicírculo de naturaleza capacitiva. Éste no está completamente resuelto en el diagrama de Nyquist, pero se distingue con claridad en la representación de Bode por la aparición de un segundo máximo en las frecuencias más bajas, junto a un cambio en la dependencia del módulo de la impedancia con la frecuencia. Según Bessone et al. (4) este arco podría estar relacionado con los procesos de adsorción de cloruros en los defectos de la capa de alúmina por donde se inicia el ataque. Por su parte, Brett et al. (5) sugieren que el mecanismo de corrosión no sólo tiene lugar a través de
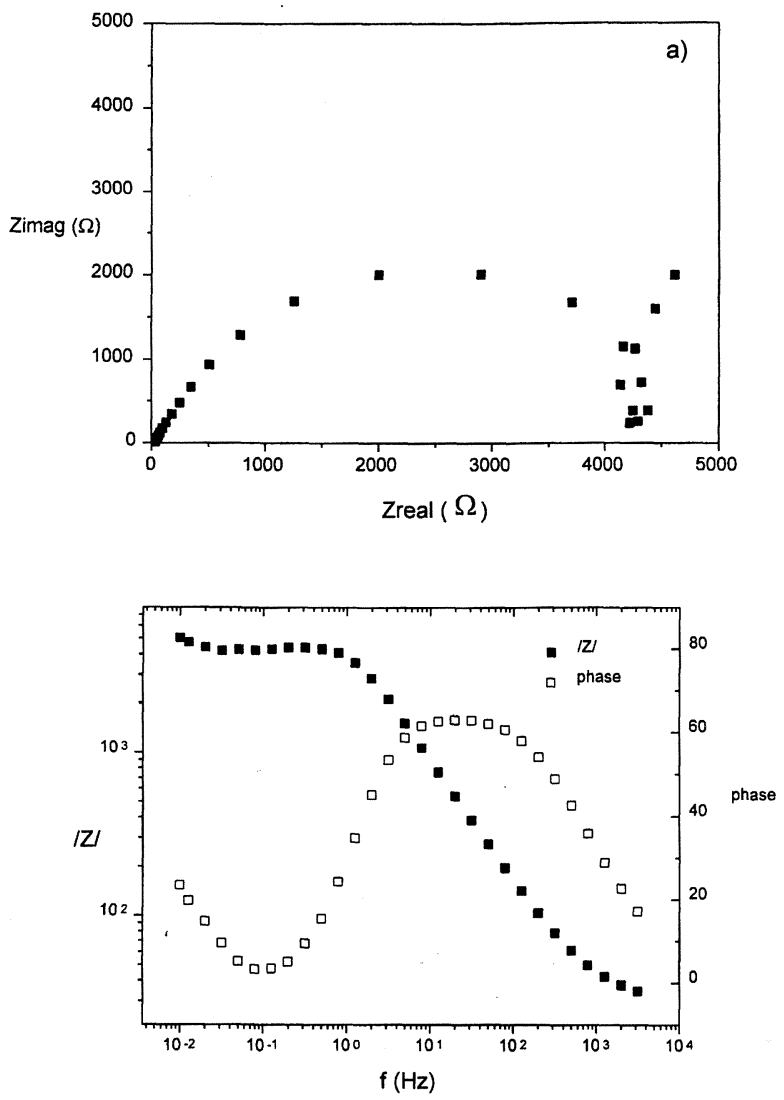

Fig. 3.- Diagramas de $a$ ) Nyquist y $b$ ) Bode, correspondientes a $100 \mathrm{~h}$ de inmersión.

FIG. 3.- Impedance diagram a) Nyquist and b) Bode after $100 \mathrm{~h}$ of testing.

los defectos de la película de óxido, sino también a través de ella por un mecanismo de migración iónica. Puesto que la capa de alúmina posee una estructura cristalina, es lógico que los defectos (vacantes) puedan tener un papel importante en el proceso difusión iónica y, por lo tanto, en la corrosión. Así, mientras que en la intercara metal-óxido la concentración de vacantes de oxígeno es mayor que en la intercara óxido-solución, en ésta la concentración de vacantes metálicas es mayor que en la primera. De esta manera, se crea un gradiente de concentración de vacantes que promueve el crecimiento de la película de óxido en la intercara metal-óxido. Cuando alcanzan la intercara más externa, los iones metálicos reaccionan con los iones cloruro presentes en el medio, formando sales solubles que favorecen la disolución del aluminio.

Estos complejos intermedios participan en la reacción de disolución del metal y producen un proceso de disolución multiescalonada, que queda reflejado por la aparición del rizo que se revela a partir de las $50 \mathrm{~h}$ de ensayo. 
A las $100 \mathrm{~h}$ de ensayo, el diagrama de impedancia está constituido por dos arcos capacitivos en altas y bajas frecuencias, que se conectan entre sí mediante un rizo, que parece ser el resultado de la evolución de un arco pseudoinductivo. Mientras el semicírculo correspondiente a las altas frecuencias podría ser consecuencia de la transferencia de carga y migración iónica a través de la capa de óxido, el rizo y el semicírculo de las frecuencias más bajas, estarían relacionados con el proceso de adsorción de cloruros que origina un proceso de disolución multiescalonada del aluminio. Sin embargo, la aparición del segundo semicírculo a las más bajas frecuencias aún no está del todo esclarecida y varios procesos podrían ser los causantes de la aparición de dicha constante de tiempo adicional (7). De entre ellas, destacan las que lo asocian a la existencia de estados de pasividad, que no parece ser aquí el caso, o las que resaltan el efecto de la adsorción de los aniones procedentes del electrólito.

Estas respuestas de la impedancia se pueden simular mediante un circuito equivalente, RC, conectado en paralelo con otro $\mathrm{RaCa}$, como el de la figura 4, donde $\mathrm{Ra}$ y $\mathrm{Ca}$ son la resistencia y la capacidad de las especies adsorbidas. La determinación de cada uno de los parámetros empleados, se realizó empleando elementos de fase constante. Los valores obtenidos para cada uno de ellos se recogen en la tabla I. Los valores de la capacidad asociada a la doble capa y a la resistencia de corrosión no cambian en el período de ensayo. Esto parece indicar que, aunque el material está siendo atacado por el medio, el ataque no es consecuencia del adelgazamiento de la película de óxido, sino que ocurre como un proceso de transferencia de carga a través de los defectos y de película protectora.

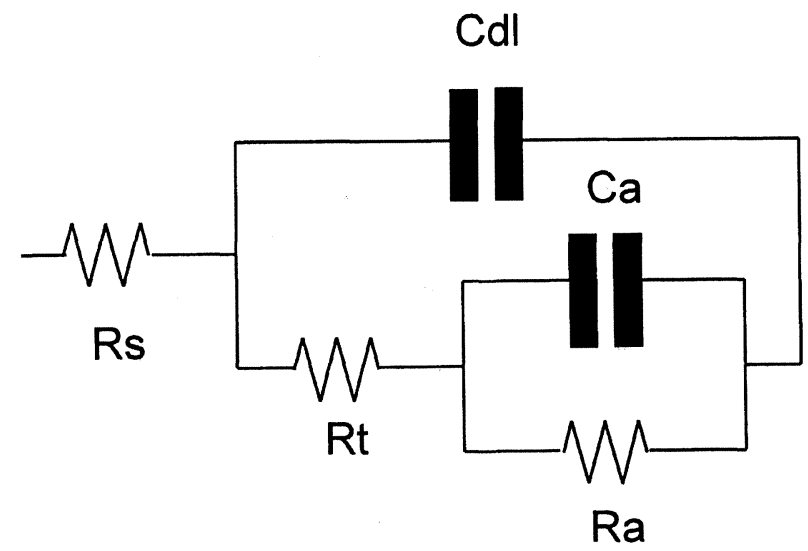

FIG. 4.- Circuito equivalente empleado para la simulación del comportamiento de la aleación 8090 en $\mathrm{NaCl} 0,06 M$.

FIG. 4.- Equivalent circuit for modelling the 8090 alloy behaviour in $\mathrm{NaCl}$ 0,06 M solution.
TABLA I.- Valores de los parámetros electroquímicos obtenidos por simulación

TABLE I.- Electrochemical parameters obtained after simulation

\begin{tabular}{|l|c|c|}
\hline & $\begin{array}{c}\text { Hasta } \\
50 \mathrm{~h}\end{array}$ & $100 \mathrm{~h}$ \\
\hline $\mathrm{Cdl}$ & $2,86 \mathrm{E}-5$ & $3,46 \mathrm{E}-5$ \\
$n$ & 0,85 & 0,81 \\
$\mathrm{Rt}$ & 4.600 & 4.610 \\
$\mathrm{Rs}$ & 40 & 31 \\
$\mathrm{Ra}$ & -1.065 & 9.230 \\
$\mathrm{Ca}$ & $-4,69 \mathrm{E}-4$ & $7,72 \mathrm{E}-3$ \\
$n$ & 0,75 & 1 \\
\hline
\end{tabular}

\section{CONCLUSIONES}

Los iones cloruro se adsorben a través de los defectos y discontinuidades de la película natural de óxido protector formado sobre la superficie de la aleación, formando compuestos intermedios que facilitan el proceso corrosivo (disolución multiescalonada). Este fenómeno se debe a la existencia de un gradiente de vacantes en las intercaras metalóxido y óxido-electrólito, que favorecen el movimiento de los iones $\mathrm{Al}^{3+}$ y oxígeno. De este modo, cuando el aluminio alcanza la intercara óxido-solución, reacciona con los iones cloruro formando sales solubles que retiran el catión metálico y permiten que continúe el ataque sobre la aleación. Esta situación se pone de manifiesto claramente observando la evolución de los diagramas de impedancia que permiten asociar a cada respuesta del sistema una etapa diferente en el proceso corrosivo.

\section{REFERENCIAS}

(1) Fielding, P.S. y Wolf, G.J. Adv. Mater. Proc. 10, 1996: 21-23.

(2) Boukamp, B.A. Proc. 9th European Cong. Corr. Utrech, 26. Oct (1989), Conf. FU-252.

(3) Conde, A. y Damborenea, J. DE. Electrochim. Acta. 43, 1998: 849-860.

(4) Ambat, R. y DwaraKadasa, E.S. J. Appl. Electrochem. 24, 1994: 911-916.

(5) Bessone, J.B., Salinas, D.R., Mayer, C.E., Ebert, M. y LORENTEZ, W. J. Electrochem. Acta. 12, 1992: 2.283-2.290.

(6) Brett, C.M., Gomes, I.A.R. y Martins, J.P.S. J. Appl. Electrochem. 24, 1994: 1.158-1.163.

(7) De Witt, J.H.W. y Lenderink, H.W. Electrochim. Acta, 41, 1996: 1.111-1.119. 